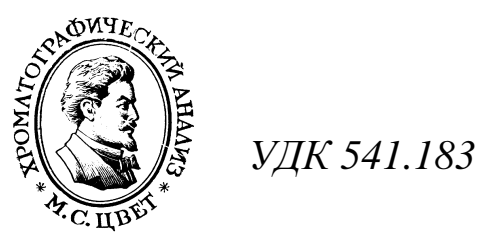

\title{
Многослойная адсорбция газов и паров на активированных углях, цеолитах и макропористых адсорбентах
}

\author{
Жданов А.А. \\ 165651, г. Коряжма, Архангельской обл., пр.им. М.В.Ломоносова, д.7, корп.3, кв 55.
}

Поступила в редакцию 6.03.2018 г.

DOI: https://doi.org/10.17308/sorpchrom.2019.19/654

В настоящее время для количественного описания изотерм адсорбции предложено большое количество уравнений. В частности, для описания изотерм адсорбции на макропористых адсорбентах используется уравнение БЭТ (или его модификации), для описания изотерм адсорбции на микропористых адсорбентах используется уравнение теории ТОЗМ, для описания изотерм адсорбции на цеолитах нет общепризнанного уравнения. В данной работе предложена концепция многослойной адсорбции и соответствующее ей уравнение изотермы адсорбции, позволяющее количественно описывать все типы изотерм адсорбции по классификации С.Брунауэра.

Ключевые слова: многослойная адсорбция, изотермы адсорбции, цеолиты, микро- и макропористые адсорбенты.

\section{Multilayered adsorption of gases and vapores on acti- vated carbons, zeolites and macroporous adsorbents}

\author{
Zhdanov A.A. \\ 165651, Koryazhma, Arkhangelsk region, pr. MV Lomonosov, 7, block 3,kv 55.
}

At present, a large number of equations have been proposed for quantitative description of adsorption isotherms for gases and vapors on solid adsorbents, which were obtained by the authors on the basis of various theoretical concepts. In particular, the equation of the theory of polymolecular adsorption of BET and its modification, proposed by GL Aranovich, and also the equations of the theory of volume filling of micropores, developed under MM Dubinin's direction, are widely used. When adsorbing gases and vapors on zeolites, it is also proposed to use equations obtained on the basis of stoichiometric and osmotic adsorption theories. It should be noted that the generally recognized equation of the adsorption isotherm, which is applicable for the quantitative description of all types of adsorption isotherms according to $\mathrm{S}$. Brunauer's classification, does not yet exist for all types of adsorbents. In this paper, an attempt is made to propose such a theory and the equation of the adsorption isotherm corresponding to this theory.

In accordance with the proposed concept of multilayer adsorption, several layers of adsorbent can be simultaneously adsorbed on the surface of the adsorbent. In the first adsorption layer, each adsorbent molecule interacts with (n) adsorption centers of the surface to form (n) «adsorbent-adsorbate» adsorption complexes. This equilibrium process is characterized by a constant of adsorption equilibrium (Kp), which enters into the three-constant equation of the adsorption isotherm. The third constant in the adsorption isotherm equation $(\mathrm{am})$ characterizes the capacity of the adsorbent layer. The surface of the adsorbent with adsorption 
complexes «adsorbent-adsorbate» is able to interact with molecules of the next adsorbent layer to form adsorption complexes «adsorbent/adsorbate-adsorbate». This equilibrium process is characterized by its equilibrium constant. Based on the range of van der Waals forces, due to which physical adsorption takes place, it can be assumed that the number of adsorption layers can not be more than three. The amount of adsorption will be equal to the sum of the adsorption of all adsorption layers at a given equilibrium pressure of the adsorbent. The coefficients in the equation of the adsorption isotherm for each adsorption layer are uniquely determined from the experimental data by the method of successive approximations under the condition that the isotherm is linear in the Cartesian coordinate system.

This paper shows the applicability of the proposed adsorption isotherm equation to describe the experimental adsorption isotherms on various types of adsorbents published in the literature. In particular, adsorption on microporous adsorbents - propane on nuksite at $333 \mathrm{~K}$, adsorption on macroporous adsorbents benzene on graphite black at $303 \mathrm{~K}$, adsorption on zeolites - xenon on $\mathrm{NaX}$ at $170 \mathrm{~K}$. It is shown that the experimental isotherms are completely satisfactorily described by the proposed equation. In this case, the adsorption of propane on the nuxite occurs in one layer, benzene on graphitized soot in two layers, and xenon on $\mathrm{NaX}$ zeolite in three layers. It is also shown that upon adsorption on a macroporous adsorbent, capillary condensation of the adsorbent occurs. To calculate the amount of condensed adsorption, an equation similar to the adsorption isotherm equation is proposed. The paper concludes that the proposed equation of the adsorption isotherm is applicable for describing all types of adsorption isotherms according to S. Brunauer classification in the entire range of equilibrium partial pressures of adsorbents. It is applicable for adsorption on micro-, macroporous adsorbents and zeolites. It is shown that the number of adsorption layers can be no more than three. Thus, the proposed concept of multilayer adsorption and the corresponding equation of the adsorption isotherm make it possible to satisfactorily describe all types of experimental adsorption isotherms according to S. Brunauer's classification on all types of adsorbents in the entire range of equilibrium partial pressures of the adsorbent.

Keywords: multilayer adsorption, adsorption isotherms, zeolites, micro- and macroporous adsorbents.

\section{Введение}

В работе [1] представлен вывод уравнения изотермы адсорбции в соответствии с представлениями теории адсорбционного равновесия, основанной на термодинамике вакансионных растворов. Уравнение для неидеального вакансионного раствора имеет следующий вид:

$$
a=a_{m} \beta p^{1 / g} /\left(1+\beta p^{1 / g}\right),
$$

где $a$ - адсорбция при равновесном давлении $p, a_{m}-$ предельная адсорбция при $p \rightarrow$ $\infty, \beta$ - постоянная, $g$ - осмотический коэффициент, зависящий от температуры.

Для идеального вакансионного раствора уравнение изотермы адсорбции (1) преобразуется в уравнение Лэнгмюра.

Как отмечено в [1] уравнение вида (1) неоднократно предлагалось рядом авторов в качестве уравнения изотермы адсорбции с различной интерпретацией трех констант, входящих в уравнение, в частности, в 1969 г., Кисаровым В.М. В работе [2] это уравнение рассматривается им как полуэмпирическое и записывается в виде:

$$
a=A B\left(p / p_{s}\right)^{n} /\left(1+B\left(p / p_{s}\right)^{n}\right)
$$

где $a$ - адсорбция при равновесном давлении $p, p_{s}$ - давление насыщенного пара, $A=W / V(V-$ объем пара или газа в адсорбированном состоянии, $W$ - объем сорбционного пространства), $B$ - постоянная величина, $n=k T / \beta$, где $\beta$ - коэффициент аффинности.

В работе [2] отмечается, что уравнение (2) вполне удовлетворительно описывает большое количество экспериментальных данных по статике адсорбции газов и паров на активированных углях, силикагелях и цеолитах в широком интервале относительных равновесных концентраций и температур. При $n=1$ это уравнение также преобразуется в уравнение Лэнгмюра. 
Одним из последних авторов, предложивших трехконстантное уравнение вида (1) в качестве уравнения изотермы адсорбции в 1983 г., был Н.Т.Селиванов [3,4]. Уравнение имеет вид:

$$
\begin{aligned}
& a_{p}=\left(a_{m}-a_{p}\right) K_{p} P_{p}{ }^{n} \text { или } \\
a_{p}= & a_{m} K_{p} P_{p}{ }^{n} /\left(1+K_{p} P_{p}{ }^{n}\right),
\end{aligned}
$$

где $a_{p}$ - количество адсорбированного вещества при его равновесном давлении в газовой фазе $P_{p}, a_{m}$ - количество адсорбированного вещества при полном заполнении слоя адсорбента, $K_{p}$ - константа равновесия процесса адсорбция - десорбция, $n-$ молекулярность, или порядок адсорбции по адсорбируемому веществу. Вместо равновесного давления адсорбтива можно пользоваться его относительной величиной $\left(P_{p} / P_{s}\right)$, где $P_{s}$ - давление насыщенного пара адсорбтива или мольной концентрацией адсорбтива в газовой (паровой) фазе $C_{p}$.

Уравнение (3) позиционируется авторами [3,4] как общее уравнение изотермы адсорбции, позволяющее количественно описать все пять типов изотерм адсорбции по классификации Брунауэра [5]. При адсорбции на однороднопористых адсорбентах при значениях $n \leq 1$ уравнение (3) описывает I тип изотермы адсорбции, при значениях $n>1$ - V тип изотермы адсорбции. При адсорбции на разнороднопористых адсорбентах или на адсорбентах с центрами адсорбции разной активности будет иметь место изотерма типа IV. В этом случае происходит двухслойная адсорбция. Константы уравнения (3) для каждого из адсорбционных слоев различны. Константа $n$ для одного из слоев будет $n \leq 1$, а для другого слоя $n>1$. Поэтому изотерма типа IV опишется как сумма изотерм I и V типов. Изотерма типа II - частный случай изотермы типа IV, а изотерма типа III - частный случай изотермы типа V.

В настоящее время для количественного описания изотерм адсорбции газов и паров на твердых адсорбентах широко используются уравнение теории полимолекулярной адсорбции БЭТ и его модификация, предложенная Г.Л.Арановичем, уравнения теории объемного заполнения микропор, разработанной под руководством М.М.Дубинина, а также уравнения, полученные на основе стехиометрической и осмотической теорий адсорбции. Следует отметить, что общепризнанного уравнения изотермы адсорбции, применимого для количественного описания всех типов изотерм адсорбции по классификации Брунауэра, для всех типов адсорбентов, пока не существует. В этой связи разработка единой теории адсорбции, применимой для количественного описания изотерм адсорбции на всех типах адсорбентов, является актуальной. В данной работе предпринята попытка предложить концепцию многослойной адсорбции и соответствующее ей уравнение изотермы адсорбции, которое удовлетворяло бы этим требованиям.

\section{Теоретическая часть}

В свете современных представлений адсорбцию можно представить как обратимую квазихимическую реакцию между адсорбционными центрами адсорбента и молекулами адсорбтива с образованием адсорбционных комплексов «адсорбентадсорбат». При этом, в случае однослойной адсорбции, $n$ свободных адсорбционных центров адсорбента $(\mathrm{R})$ взаимодействует с одной молекулой адсорбтива (A) с образованием $n$ адсорбционных комплексов «адсорбент-адсорбат» (R-A). Константа равновесия этой квазихимической реакции запишется, как:

$$
K_{p}=[R-A]^{n} /\left([R]^{n}[A]\right)
$$

Из уравнения (4), обозначив $[R-A]=a_{p},[R]=\left(a_{m}-a_{p}\right),[A]=P_{p}$, получим уравнение, которое будет описывать изотерму адсорбции слоя адсорбата: 


$$
\begin{gathered}
a_{p}^{n}=\left(a_{m}-a_{p}\right)^{n} K_{p} P_{p} \quad \text { или после небольших преобразований } \\
a_{p}=a_{m} K_{p}^{1 / n} P_{p}^{1 / n} /\left(1+K_{p}^{1 / n} P_{p}^{1 / n}\right),
\end{gathered}
$$

где $a_{p}$ - количество адсорбированного вещества при его равновесном давлении в газовой фазе $P_{p}, a_{m}$ - количество адсорбированного вещества при полном заполнении слоя адсорбента, $K_{p}$ - константа равновесия процесса адсорбция - десорбция, $n-$ молекулярность, или порядок адсорбции по адсорбенту. При $n=1$ это уравнение преобразуется в уравнение Лэнгмюра.

Поверхность адсорбента с адсорбционными комплексами «адсорбентадсорбат» способна вновь взаимодействовать с молекулами адсорбтива с образованием следующего слоя адсорбционных комплексов «адсорбент-адсорбат/адсорбат». Изотерма адсорбции второго и последующих слоев адсорбата также может быть описана уравнением (5). Формирование второго и последующих слоев начинается одновременно с формированием первого слоя адсорбата, как и в теории БЭТ. По мере роста равновесного парциального давления адсорбтива растет и величина адсорбции в каждом слое адсорбата (это можно видеть и на рисунках №№ 1-3). Суммарная адсорбция всех слоев адсорбата, при условии, что адсорбция всех слоев адсорбата происходит одновременно, запишется, как:

$$
a_{p}=\sum_{i=1}^{j} a_{p i}=\sum_{i=1}^{j} a_{m i} K_{p i}^{1 / n i} P_{p}^{1 / n i} /\left(1+K_{p i}^{1 / n i} P_{p}^{1 / n i}\right)
$$

В этом уравнении вместо абсолютных значений равновесного давления адсорбтива $\left(P_{p}\right)$ можно использовать и относительные $\left(P_{p} / P_{s}\right)$. При этом изменится размерность и численные значения констант равновесия $\left(K_{p i}\right)$. Для каждого из слоев адсорбата на поверхности адсорбента значения констант в уравнении (6) различны. С учетом радиуса действия сил Ван-дер-Ваальса, за счет которых и происходит физическая адсорбция, можно предположить, что слоев адсорбата на поверхности адсорбента может быть не более трех $(i \leq 3)$.

Значения констант в уравнении (6) для каждого слоя адсорбата однозначно определяются из экспериментальных данных методом последовательных приближений из условия линейности изотермы адсорбции в координатах $a_{p}-a_{p} /\left(P_{p}\right)^{1 / n}$. Линейная форма изотермы адсорбции для одного слоя адсорбата запишется как:

$$
a_{p}=a_{m}-\frac{1}{K_{p}^{1 / n}} * \frac{a_{p}}{P_{p}^{1 / n}}
$$

\section{Эксперимент}

В данной работе использованы экспериментальные данные, опубликованные в литературе, в частности, экспериментальная изотерма адсорбции пропана на нуксите при 333 К [6], ксенона на цеолите $\mathrm{NaX}$ при 170 К [7], бензола на графитированной саже при 303 К [8]. Экспериментальные данные приведены в таблице 1.

Таблица 1. Экспериментальные изотермы адсорбции

\begin{tabular}{|c|c|c|c|c|c|}
\hline \multicolumn{2}{|c|}{$\begin{array}{c}\text { Адсорбция пропана } \\
\text { на нуксите, } 333 \mathrm{~K} .\end{array}$} & \multicolumn{2}{|c|}{$\begin{array}{c}\text { Адсорбция ксенона } \\
\text { на цеолите } \mathrm{NaX}, 170 \mathrm{~K} .\end{array}$} & \multicolumn{2}{|c|}{$\begin{array}{c}\text { Адсорбция бензола } \\
\text { а графитированной саже, } 303 \mathrm{~K} .\end{array}$} \\
\hline \multicolumn{2}{|c|}{$\mathrm{P}_{\mathrm{s}}=2.16 \mathrm{MПа}$} & \multicolumn{2}{|c|}{$\mathrm{P}_{\mathrm{s}}=135$ кПа } & \multicolumn{2}{c|}{$\mathrm{P}_{\mathrm{s}}=16136$ Па } \\
\hline$P_{p} / P_{s}$ & $\begin{array}{c}a \text { эксn., } \\
\text { моль/кг }\end{array}$ & $P_{p}$, КПа & $\begin{array}{c}a \text { эксn., } \\
\text { моль/кг }\end{array}$ & $P_{p} / P_{s}$ & $\begin{array}{c}a \text { эксn., } \\
\text { моль/кг }\end{array}$ \\
\hline 1 & 2 & 3 & 4 & 5 & 6 \\
\hline 0.000164 & 0.316 & 0.000486 & 0.054 & 0.0000033 & 0.0011 \\
\hline
\end{tabular}




\begin{tabular}{|c|c|c|c|c|c|}
\hline 1 & 2 & 3 & 4 & 5 & 6 \\
\hline 0.00274 & 1.222 & 0.001026 & 0.12 & 0.000129 & 0.0042 \\
\hline 0.00483 & 1.56 & 0.0013365 & 0.143 & 0.000778 & 0.015 \\
\hline 0.00807 & 1.923 & 0.001755 & 0.213 & 0.001046 & 0.0205 \\
\hline 0.0139 & 2.32 & 0.00243 & 0.276 & 0.00166 & 0.0292 \\
\hline 0.0209 & 2.626 & 0.00378 & 0.425 & 0.00208 & 0.035 \\
\hline 0.0264 & 2.793 & 0.00567 & 0.594 & 0.00258 & 0.0445 \\
\hline 0.0426 & 3.151 & 0.00702 & 0.676 & 0.00404 & 0.0588 \\
\hline 0.0444 & 3.168 & 0.01134 & 0.931 & 0.0045 & 0.0647 \\
\hline 0.0487 & 3.227 & 0.01485 & 1.09 & 0.00591 & 0.0833 \\
\hline 0.0516 & 3.27 & 0.0216 & 1.33 & 0.00914 & 0.1143 \\
\hline 0.0556 & 3.318 & 0.04455 & 1.93 & 0.01157 & 0.1353 \\
\hline 0.0659 & 3.445 & 0.06075 & 2.28 & 0.02138 & 0.1934 \\
\hline 0.0969 & 3.69 & 0.0729 & 2.46 & 0.0332 & 0.2408 \\
\hline 0.107 & 3.756 & 0.09315 & 2.83 & 0.0355 & 0.2493 \\
\hline 0.123 & 3.842 & 0.1107 & 3.04 & 0.0415 & 0.2645 \\
\hline 0.172 & 4.04 & 0.162 & 3.64 & 0.05198 & 0.2834 \\
\hline 0.207 & 4.148 & 0.243 & 4.21 & 0.0695 & 0.3057 \\
\hline 0.24 & 4.236 & 0.4185 & 4.76 & 0.0973 & 0.3276 \\
\hline 0.278 & 4.326 & 0.5535 & 4.86 & 0.1203 & 0.3455 \\
\hline 0.31 & 4.325 & 0.9315 & 5.07 & 0.1348 & 0.3548 \\
\hline 0.35 & 4.307 & 2.295 & 5.38 & 0.1421 & 0.3618 \\
\hline 0.4 & 4.348 & 2.97 & 5.42 & 0.1614 & 0.3726 \\
\hline 0.45 & 4.379 & 57.915 & 6.34 & 0.1802 & 0.3773 \\
\hline 0.55 & 4.423 & 72.495 & 6.49 & 0.2027 & 0.3897 \\
\hline 0.6 & 4.438 & 75.06 & 6.6 & 0.2334 & 0.4083 \\
\hline 0.65 & 4.45 & 84.375 & 6.72 & 0.3158 & 0.4379 \\
\hline 0.7 & 4.459 & 92.205 & 6.8 & 0.4759 & 0.5384 \\
\hline 0.85 & 4.476 & & & 0.737 & 0.9048 \\
\hline 0.9 & 4.478 & & & 0.7966 & 1.07546 \\
\hline & & & & 0.8356 & 1.3618 \\
\hline & & & & 0.8959 & 2.0279 \\
\hline & & & & 0.9349 & 3.5021 \\
\hline & & & & & \\
\hline
\end{tabular}

\section{Обсуждение результатов}

В работе [6] представлен расчет величин адсорбции на однороднопористых активных углях по наиболее часто используемым уравнениям. В работе сделано заключение, что для описания изотерм адсорбции на однороднопористых активных углях лучше других уравнений подходит уравнение ТОЗМ Дубинина-Радушкевича. На примере адсорбции пропана на нуксите при 333 К покажем применимость уравнения (6) для описания данной экспериментальной изотермы. Результаты представлены на рисунке 1. Как можно видеть из полученных результатов, уравнение (6) вполне удовлетворительно описывает экспериментальную изотерму во всей области равновесных давлений адсорбтива. Значения констант уравнения (6) приведены в таблице 2. Адсорбция на однороднопористых адсорбентах - однослойная. Изотерма адсорбции пропана на нуксите относится к типу I по классификации Брунауэра.

В работе [7] представлен расчет величин адсорбции на цеолитах по наиболее часто используемым уравнениям. В работе отмечается, что в литературе нет общепризнанного уравнения для описания изотерм адсорбции газов и паров на различных цеолитах. На примере адсорбции ксенона на цеолите $\mathrm{NaX}$ при 170 К покажем применимость уравнения (6) для описания данной экспериментальной изотермы. Результаты представлены на рис. 2. Как можно видеть из полученных результатов 
уравнение (6) вполне удовлетворительно описывает экспериментальную изотерму адсорбции на цеолите во всей области равновесных парциальных давлений адсорбтива. Значения констант уравнения (6) приведены в таблице 2. Адсорбция ксенона на цеолите $\mathrm{NaX}$ протекает в три слоя. Изотерма адсорбции ксенона на цеолите $\mathrm{NaX}$ относится к типу IV по классификации Брунауэра. Уравнение (6) точнее описывает изотерму адсорбции ксенона на цеолите, чем, например, уравнение ТОЗМ Дубинина-Астахова (значения коэффициентов этого уравнения: $n=3, E_{0}=10739$ дж/моль, $a_{0}=6.26$ моль/кг).

Таблица 2. Значения коэффициентов уравнения (6), использованные в данной работе.

\begin{tabular}{|c|c|c|c|c|c|c|c|c|c|}
\hline $\begin{array}{c}\text { Адсорб- } \\
\text { ционная } \\
\text { система }\end{array}$ & $1 / n_{1^{-}}$ & $\begin{array}{c}a_{m l,} \\
\text { моль/ кг }\end{array}$ & $\begin{array}{c}\left(K_{p}\right)_{1}, \\
-/(\kappa \Pi a)^{-1}\end{array}$ & $1 / n_{2}$ & $\begin{array}{c}a_{m 2}, \\
\text { моль/кг }\end{array}$ & $\begin{array}{c}\left(K_{p}\right)_{2}, \\
-/(\kappa \Pi a)^{-1}\end{array}$ & $1 / n_{3^{-}}$ & $\begin{array}{c}a_{m 3}, \\
\text { моль/кг }\end{array}$ & $\begin{array}{c}\left(K_{p}\right)_{3}, \\
-/(\kappa \Pi a)^{-1}\end{array}$ \\
\hline $\begin{array}{c}\text { пропан - } \\
\text { нуксит } \\
333 К\end{array}$ & 0.58 & 5.08 & $53.9 /-$ & - & - & - & - & - & - \\
\hline $\begin{array}{c}\text { ксенон- } \\
\text { цеолит } \\
\text { КаХ 170К }\end{array}$ & 1.1 & 2.03 & $-/ 77.83$ & 1.8 & 3.16 & $-/ 6.88$ & 1 & 4.31 & $-/ 0.0063$ \\
\hline $\begin{array}{c}\text { бензол - } \\
\text { графитир. } \\
\text { сажа 303К }\end{array}$ & 0.55 & 0.0073 & $13247 /-$ & 1.05 & 0.39 & $0.408 /-$ & - & - & - \\
\hline
\end{tabular}

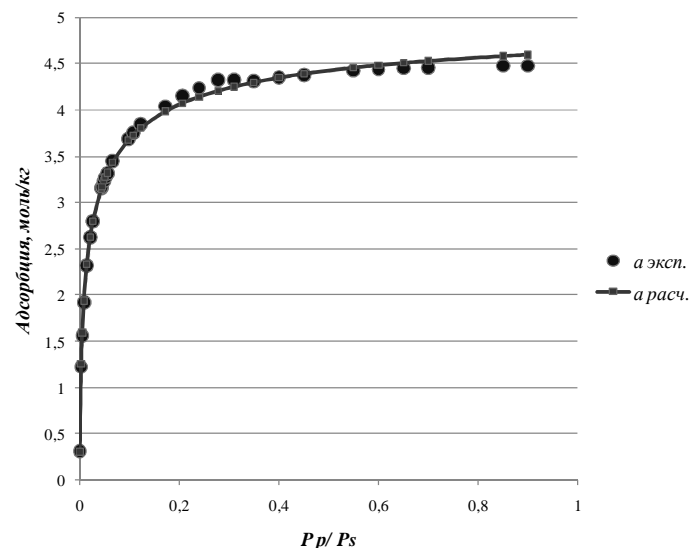

Рис. 1. Адсорбция пропана на нуксите, Т 333К, Ps=2.16 МПа.

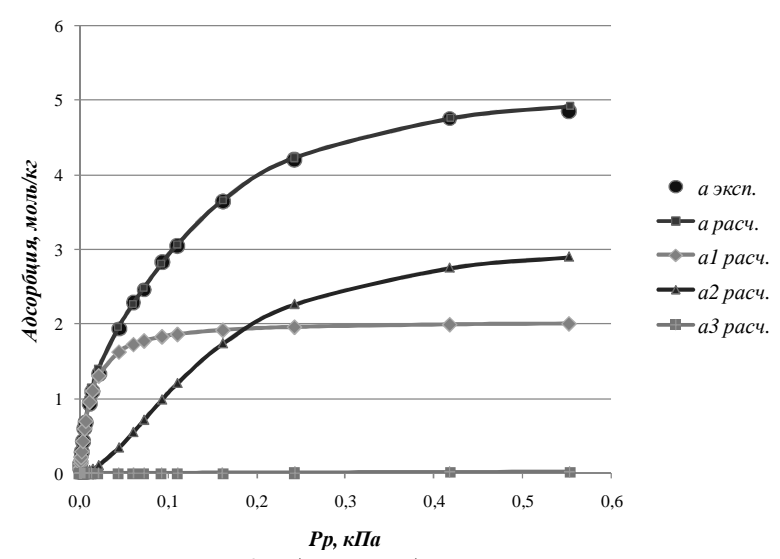

Рис. 2. Адсорбция ксенона на цеолите $\mathrm{NaX}$. T 170K, Ps=135 кПа.

В работе [8] отмечается, что для описания адсорбционных равновесий при адсорбции на макропористых адсорбентах чаще всего используется уравнение полимолекулярной адсорбции БЭТ. Отмечается также, что применимость этого уравнения ограничена интервалом равновесных давлений (0.03-0.3) $P_{p}$. Модификация этого уравнения Арановичем Г.Л. позволяет увеличить этот интервал до (0.03-0.7) $P_{p}$. В качестве примера приводится описание этими уравнениями экспериментальных изотерм адсорбции бензола на графитированной саже. Покажем применимость предлагаемого уравнения (6) для описания данной изотермы. Экспериментальные данные взяты в [9], результаты представлены на рис. 3. Значения коэффициентов уравнения (6) представлены в таблице 2. После расчета величин адсорбции $\left(a_{1}\right.$ pacч.) и $\left(a_{2}\right.$ расч.) в первых двух адсорбционных слоях по уравнению (6) и вычета этих значений из экспериментальных значений получим кривую $a_{3}$ эксn. $=f\left(P_{p} / P_{s}\right)$, отображающую адсорбцию в третьем слое, которая изображена на рис. 4. Здесь $a_{3}$ эксn. $=(a$ 
эксn. - $a_{1}$ расч.- $a_{2}$ расч.). Эта кривая уходит в бесконечность при приближении $\left(P_{p} / P_{s}\right) \kappa 1$. Эта кривая не описывается уравнением (6). Адсорбция не может быть бесконечной, поэтому в данном случае наблюдается не адсорбция, а конденсация адсорбтива в третьем слое. В координатах $\left(P_{p} / P_{s}\right)-a_{3}$ эксn. эта кривая изображена на рисунке 5 , значения $\left(P_{p} / P_{s}\right)$ на этой кривой асимптотически приближается к 1 . Для описания получаемой сингулярности предлагаю использовать следующее уравнение: $\left(P_{p} / P_{s}\right)=\left(1-\left(P_{p} / P_{s}\right)\right) \cdot K \cdot a$ конденс., или $P_{p}=\left(P_{s}-P_{p}\right) \cdot K \cdot a$ конденс., если пользоваться абсолютными, а не относительными значениями равновесного давления адсорбтива. Отсюда получаем уравнение для определения количества сконденсировавшегося адсорбтива в третьем слое:

$$
\left.a_{\text {кондес. }}=\left(P_{p} / P_{s}\right) / K\left(1-\left(P_{p} / P_{s}\right)\right)\right] \text { или } a_{\text {конденс. }}=P_{p} /\left[K\left(P_{s}-P_{p}\right)\right],
$$

если пользоваться абсолютными, а не относительными значениями равновесного давления адсорбтива. Параметр $K$ определяется из линейной формы кривой $a_{3}$ эксn. $=f\left(P_{p} / P_{s}\right)$ в координатах $\left(P_{p} / P_{s}\right)-\left(P_{p} / P_{s}\right) / a_{3}$ эксn. В данном случае в ходе обработки экспериментальных данных получено значение $K=5.43$ кг/моль. Как можно видеть из полученных результатов уравнение (6) в сочетании с уравнением (8) вполне удовлетворительно описывает экспериментальную изотерму адсорбции на макропористых адсорбентах во всей области равновесных давлений. Таким образом, адсорбция бензола на графитированной саже протекает в два слоя, а в третьем слое наблюдается конденсация адсорбтива на поверхности «адсорбент-адсорбат/адсорбат». Изотерма адсорбции бензола на графитированной саже относится к типу II по классификации Брунауэра.

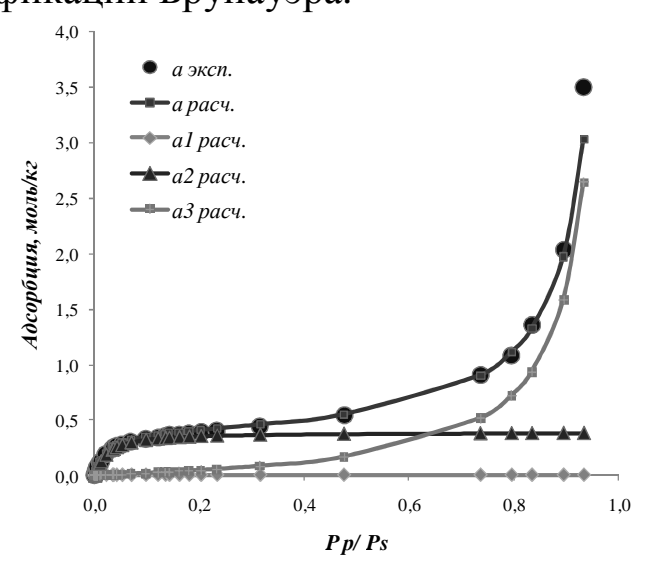

Рис.3. Адсорбция бензола на графитированной саже. Т $303 \mathrm{~K}$, Ps $=16136$ Па.

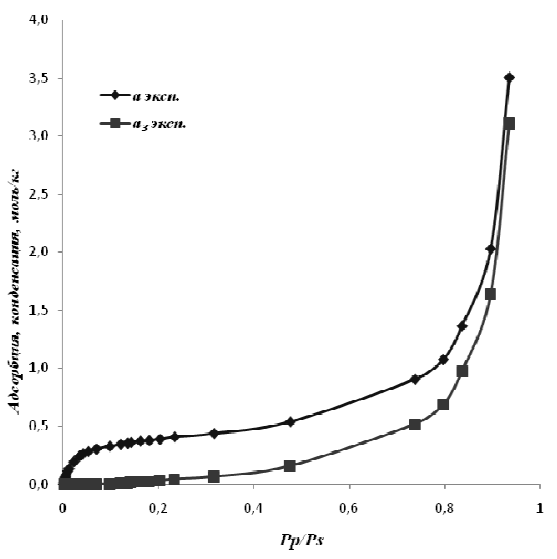

Рис.4.Адсорбция и конденсация бензола на графитированной саже $303 \mathrm{~K}, \mathrm{Ps}=16136$ Па

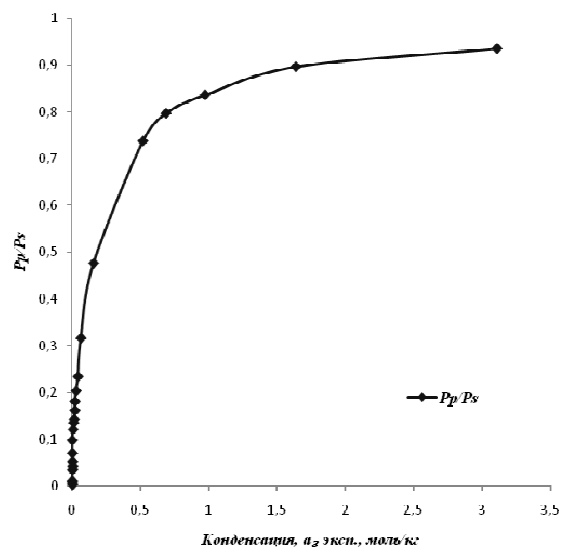

Рис. 5. Конденсация бензола на графитированной саже 303 K, Ps=16136 Па. 
Методика определения коэффициентов уравнения (6). Значения констант уравнения (6) для каждого слоя адсорбата однозначно определяются из экспериментальных данных методом последовательных приближений из условия линейности изотермы адсорбции в координатах $a_{p}-a_{p} /\left(P_{p}\right)^{1 / n}$. Линейная форма изотермы адсорбции для одного слоя адсорбата представлена уравнением (7). Покажем нахождение констант уравнения (6) на примере адсорбции пропана на нуксите при 333 К. На рисунке №6 представлены экспериментальные данные в координатах $a_{p}-a_{p} /\left(P_{p}\right)^{1 / n}$ при $1 / n=1$. Последовательно увеличивая $n$ приближаемся к линейной форме изотермы адсорбции, которая представлена на рисунке 7. При этом $1 / n=0.58$. На оси ординат при этом отсекается значение $a_{m}=5.078$ моль/кг, из тангенса угла наклона прямой определяется значение константы равновесия $\left(K_{p}\right)^{0,58}=1 / 0.099=10.1$. Отсюда $\mathrm{K}_{\mathrm{p}}=53.9$.

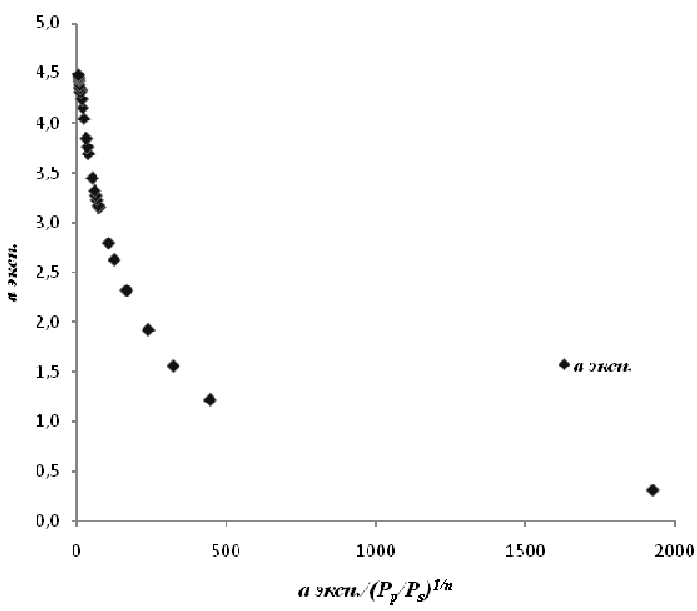

Рис. 6. Адсорбция пропана на нуксите $333 \mathrm{~K}, \mathrm{Ps}=2.16 \mathrm{MПа}, 1 / \mathrm{n}=1$

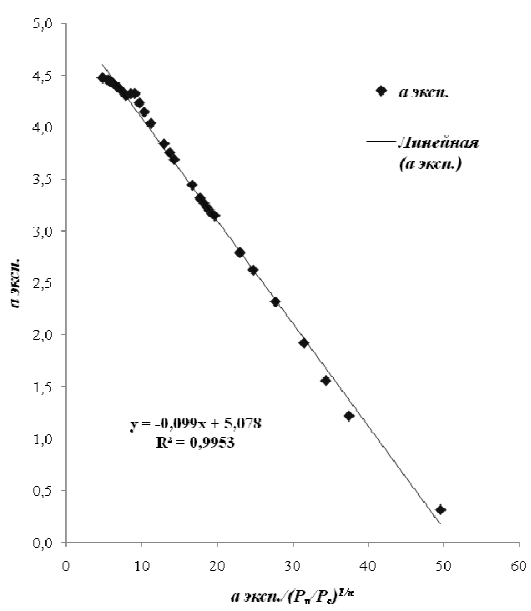

Рис. 7. Адсорбция пропана на нуксите $333 \mathrm{~K}, \mathrm{Ps}=2.16 \mathrm{MПа}, 1 / \mathrm{n}=0.58$

Из рисунка №7 видно, что все экспериментальные точки изотермы ложатся на прямую с допустимой погрешностью. В этом случае адсорбция однослойная. В случае двух и трехслойной адсорбции по начальному участку изотермы определяются константы уравнения (6) для первого слоя адсорбата и вычисляются значения величины адсорбции в первом слое $\left(a_{p}\right)_{1}$. Далее эти значения вычитаются из экспериментальных значений величины адсорбции и по оставшимся значениям величины адсорбции определяются значения констант уравнения (6) для второго и, если потребуется, то и для третьего слоя адсорбата или сконденсировавшегося адсорбтива по уравнению (8).

\section{Заключение}

Предложена концепция многослойной адсорбции и соответствующее уравнение изотермы адсорбции (6). На примерах экспериментальных литературных данных показана применимость данного уравнения для описания изотерм адсорбции на микро- и макропористых адсорбентах, а также на цеолитах. Показано, что уравнение (6) описывает все типы изотерм адсорбции по классификации С.Брунауэра во всем интервале равновесных давлений адсорбтива. При адсорбции на макропористых адсорбентах наряду с адсорбцией наблюдается также и конденсация адсорбтива, которую можно описать уравнением (8). Уравнение изотермы адсорбции (6) является универсальным и применимо для описания изотерм адсорбции всех типов на всех типах адсорбентов. 


\section{Список литературы}

1. Беринг Б.П., Серпинский В.В. // Известия АН СССР. Серия Химия. 1974. № 11. С. 2427-2440.

2. Кисаров В.М. Журнал физической химии. 1969. Т. 43. № 4. С. 1037-1039.

3. Заяц Ю.Н., Жданов А.А., Селиванов Н.Т. Журнал прикладной химии. 1983. Т. 56. № 2. С. 397-400.

4. Жданов А.А., Селиванов Н.Т. Журнал прикладной химии. 1984. Т. 57. № 8. С.18641867.

5. Брунауэр С. Адсорбция газов и паров. М. Издательство иностранной литературы. 1948. Т. 1. C. 134-208.

6. Годовиков И.А., Кузнецова Т.А., Толмачев А.М. Журнал физической химии. 2001. Т. 75. № 11. C. 2030-2036.

\section{References}

1. Bering B.P.. Serpinskiy V.V., Izvestiya AN SSSR. Seriya khimiya, 1974, No 11, pp. 24272440.

2. Kisarov V.M., Zhurnal fizicheskoy khimii, 1969, Vol. 43, No 4, pp. 1037-1039.

3. Zayats Yu.N., Zhdanov A.A., Selivanov N.T., Zhurnal prikladnoy khimii, 1983, Vol. 56, No 2, pp. 397-400.

4. Zhdanov A.A., Selivanov N.T., Zhurnal prikladnoy khimii, 1984, Vol. 57, No 8, pp.1864-1867.

5. Brunauer S. Adsorbtsiya gazov i parov. M.. Izdatelstvo inostrannoy literatury. 1948, Vol. 1, pp. 134-208.

Жданов Андрей Александрович - к.т.н., Пенсионер, 165651, Коряжма, Архангельской обл., пр.им. М.В.Ломоносова, д.7, корп.3, кв 55.
7. Толмачев А.М., Трубников О.И., Годовиков И.А., Кузнецова Т.А. Вестник Московского Университета. Серия 2. Химия. 2001. T. 42. № 4. C. 247-250.

8. Толмачев А.М. Описание адсорбционных равновесий. Сорбиионные и хроматографические прочессы. 2009. Т. 9. № 1. С. 532.

9. Толмачев А.М., Годовиков И.А., Кузнецова Т.А., Крюченкова Н.Г. и др. Банк данных адсорбция. 2015. Хим. Фак. МГУ. Режим доступа: http://www.adsbank.chem.msu.ru/ (дата обращения 15.06.2017)

6. Godovikov I.A.. Kuznetsova T.A., Zhurnal fizicheskoy khimii, 2001, Vol. 75, No 11, pp. 2030-2036.

7. Tolmachev A.M., Trubnikov O.I., Godovikov I.A., Kuznetsova T.A., Vestnik Moskovskogo Universiteta. Seriya 2. Khimiya, 2001, Vol. 42, No 4, pp. 247-250.

8. Tolmachev A.M., Sorbtsionnyye i khromatograficheskiye protsessy, 2009, Vol. 9, No 1, pp. 5-32.

9. Tolmachev A.M.. Godovikov I.A.. Kuznetsova T.A.. Kryuchenkova N.G. et al., Bank dannykh adsorbtsiya 2015. Khim. Fak. MGU. Available at: http://www.adsbank.chem.msu.ru/ (accesed 15 June 2017)

Zhdanov Andrey A. - candidate of technical sciences, pensioner, E-mail: andrey.zhdanov.51@mail.ru 